\title{
Merkel cell carcinoma. Case report
}

\author{
Richard Pinka , Martin Molitor ${ }^{b}$, Jiri Ehrmannc, Peter Tvrdy ${ }^{a}$, Petr Michla ${ }^{a}$ Jindrich Pazdera ${ }^{a}$
}

Background. Merkel cell carcinoma (MCC) is a rare aggressive skin tumour affecting mainly older and immuno suppressed individuals (see our review on MCC in this volume).

Aim. This is a case report describing our first experience with Merkel cell carcinoma in an elderly woman on chemo therapy for lymphoma and it covers the diagnosis, treatment and outcome.

Methods. We did a radical excision of the soft tissue in the tumor area in conjunction with total paroditectomy and resection of the body of the zygomatic bone by radical block neck dissection. The skin defect was reconstructed myocutaneous free flap by a plastic surgeon.

Results and Conclusion. At 12 months follow-up, the MCC had not recurred.

Key words: merkel cell carcinoma, immuno supression, diagnosis, treatment

Received: November 1, 2011; Accepted: February 28, 2012; Available online: June 1, 2012

http://dx.doi.org/10.5507/bp.2012.034

${ }^{a}$ Department of Oral and Maxillofacial Surgery, University Hospital Olomouc, Czech Republic

${ }^{b}$ Department of Plastic and Aesthetic Surgery, Faculty of Medicine and Dentistry, Palacky University Olomouc and University Hospital

Olomouc

'Department of Pathology, Faculty of Medicine and Dentistry, Palacky University Olomouc and University Hospital Olomouc

Corresponding author: Richard Pink, e-mail: richard.pink@seznam.cz

\section{CASE REPORT}

A 77 year old woman was examined at the Clinic of Oral and Maxillofacial Surgery on the recommendation of a dermatologist for a skin lesion of $10 \mathrm{~mm}$ x $10 \mathrm{~mm}$ located on the left temporal subcutaneous external corner near the left eye. The lesion clinically resembled a capillary hemangioma. In the anamnesis, the patient had been treated with CHOP (cyklofofamid - Endoxan, Adriablastin - Doxorubicin, Vincristine) myeloablative chemotherapy at the Haemato-oncology Clinic for lymphoma. Among long-term immuno-suppressive drugs given was Prednisone.

In the three weeks that elapsed between the first examination and the surgical removal, the tumor had roughly doubled in volume. Histopathological examination showed a solid tumor, located in the dermis and epidermis. The tumor was composed of cells with large round nuclei, fine chromatin and small nucleoli with high mitotic activity. The immunohistochemistry showed CK20 and NSE positivity and negativity of S100 protein. Proliferative activity assessed by the Ki-67 index was $50 \%$. The tumor lesions reached the base of the excision but were not in the lateral edges.

On the basis of the histological findings, radical surgical scar revision was done. The biopsy revealed a malignant tumor structure. The postoperative defect was reconstructed with a local flap.

On the check-up for cancer at intervals of 4 weeks, following the second surgery, we found a circumscribed, painless swelling $10 \times 10 \mathrm{~mm}$ in size in the left subcutaneous tragus. After extirpation, the lymph node was found to be infiltrated by Merkel cell carcinoma.
Examination by PET-CT showed glucose hypermetabolism in the mediastinum, lungs and spleen but not in regional lymph nodes. Compared to similar testing performed 7 months before the follow-up at the Haematooncology Clinic for malignant lymphoma, we uncovered progression to lymph nodes and spleen. The findings were discussed with the hemato-oncologist who concluded that this was a fundamental change associated with the hematological disease.

Based on the histological findings in the preauricular lymph nodes, we proceeded to do a radical excision of

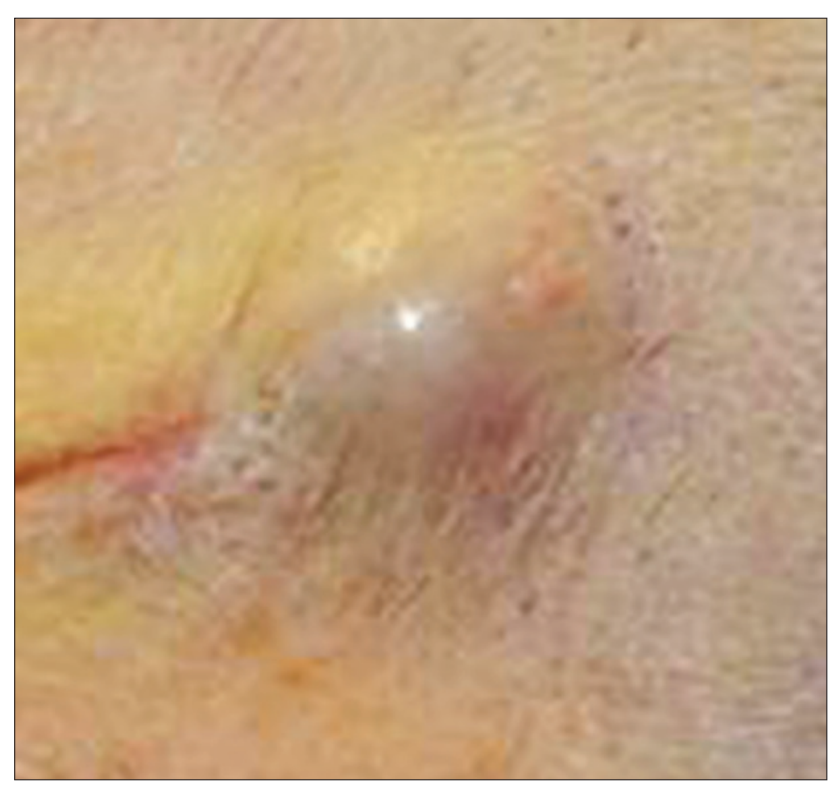

Fig. 1. Skin lesion of MCC. 


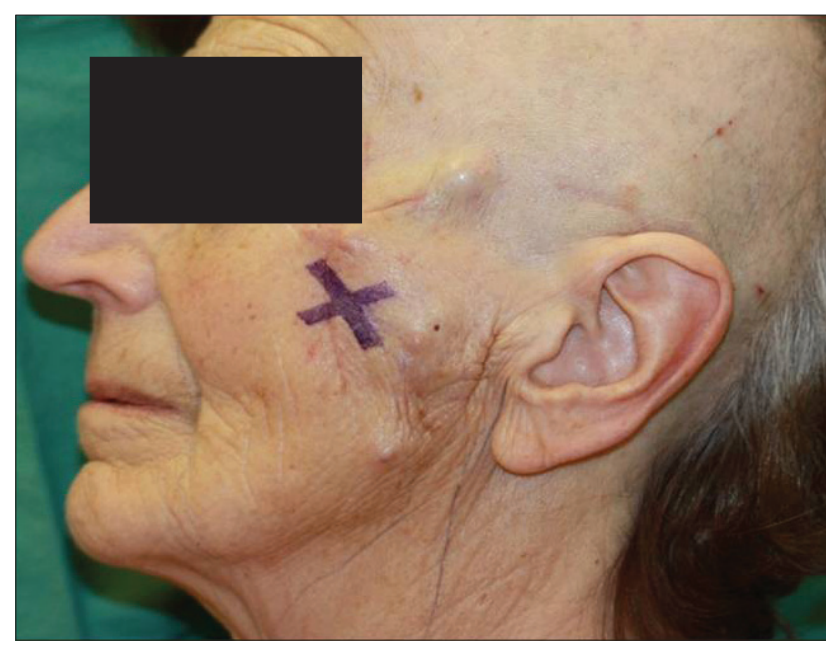

Fig. 2. Relaps of MCC in 4 weeks.

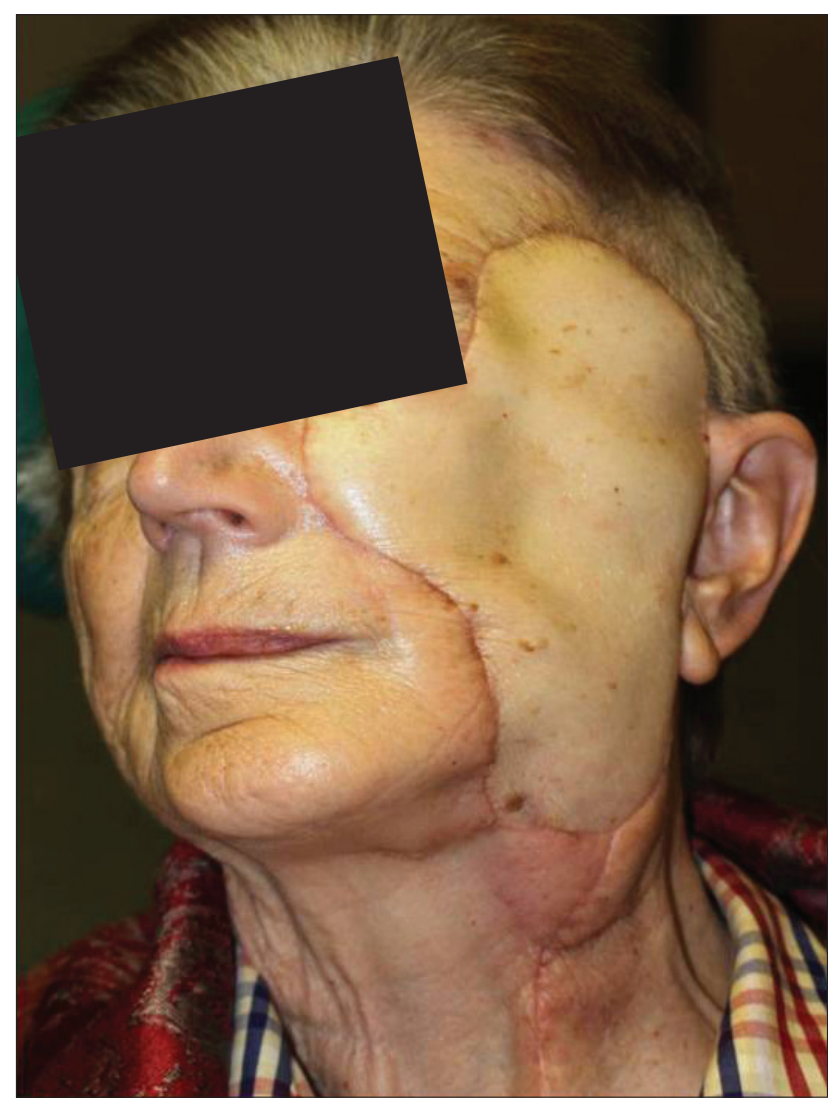

Fig. 4. Reconstruction of the defect by myocutaneous free flap (latissimus dorsi m.).

the soft tissue in the tumor area, accompanied by total paroditectomy and resection of the body of the zygomatic bone by radical block neck dissection. The skin defect was reconstructed in conjunction with a plastic surgeon with myocutaneous free flap (latissimus dorsi m.). The histology confirmed the presence of scar tissue in the tumor after primary surgery and in parotid and submandibular lymph nodes. Tumor tissue at the back edge of the specimen did not reach further than $3 \mathrm{~cm}$ from the edge of the resection. Based on the histopathological findings, the tumor was assigned to stage III malignant disease. (Positive metastatic submandibular lymph nodes).

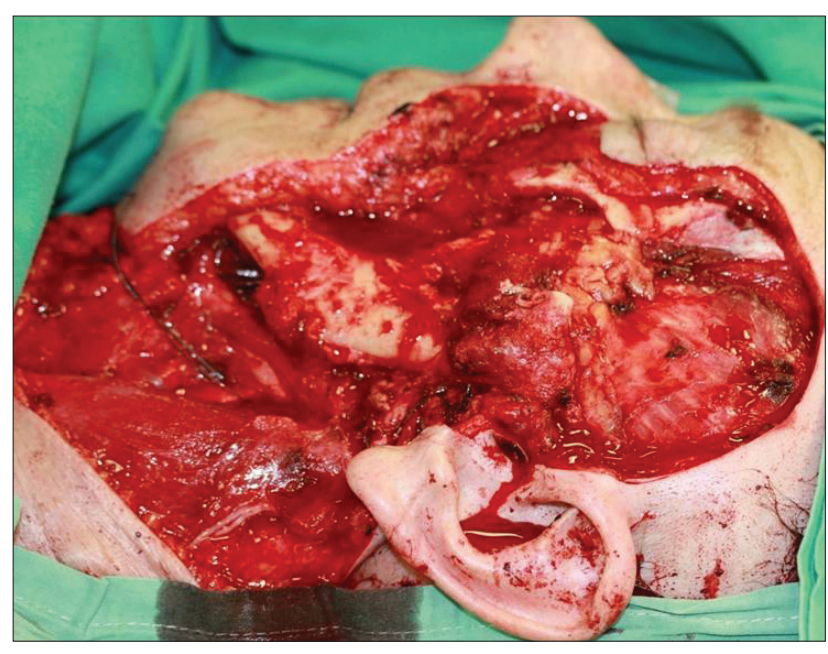

Fig. 3. Radical excision of the soft tissue in the tumor area, accompanied by total paroditectomy and resection of the body of the zygomatic bone by radical block neck dissection.

After flap engraftment and following an interval of several weeks, cancer therapy consisting of chemoradiotherapy - (DDP in a single dose of $20 \mathrm{mg} / \mathrm{m}^{2}$ weekly, RT 60 Gy over 6 weeks) was started.

Today,one year after diagnosis of MCC, the condition of the patient is stable, with no signs of loco-regional recurrence of MCC and in hematooncological terms no evidence of further progression of the malignant lymphoma.

\section{DISCUSSION}

In the present case report, we highlight a malignancy with a grave prognosis and which we had never in clinical practice encountered before.

$\mathrm{MMC}$ is a rare malignancy with an uncertain prognosis ${ }^{1}$. Owing to its uncommon occurrence and dearth of randomized studies there is no agreed and proven optimal treatment. The tumor was only recently included in the international classification of tumors ( $\mathrm{NCCN})$.

Clinical diagnosis is very difficult: the first clinical manifestation of symptoms are subtle and nonspecific. The tumor can be easily regarded as a hemangioma or other benign tumor. For these reasons, the primary surgery may not be radical enough as was the case in our patient.

MCC occurs predominantly in older people and those with weakened immune systems ${ }^{2,3}$. In addition to immune deficiency a subject of recent interest is the Merkel Cell Polyomavirus (MCPyV), which was only first described in 2008 as one of 7 known human oncogenic viruses ${ }^{4}$. However, it is now clear that the MCPyV seroprevalence among the general population is high. Its direct effect on cancer remains controversial and from a prognostic point of view, positive $\mathrm{MCPyV}$ in $\mathrm{MCC}$ is unclear. In any event; it had little relevance to our patient.

Although the general characteristics of Merkel cell carcinoma is early and rapid growth of metastases, there are publications on spontaneous regression ${ }^{5}$. Mori et al. ${ }^{6}$ 
observed high apoptotic activity of tumor in 8 cases of patients treated for MCC. Inoue et al investigated the mechanism of spontaneous regression in 7 patients (4 patients with and 3 without spontaneous regression. This study looked at a number of parameters (TUNEL index, labeling index of proliferating cell nuclear antigen (PCNA) labeling index of the bcl-2 protein expression, p53 in cells and extent of lymphocytic infiltration around tumor ${ }^{7}$. The number of T lymphocytes in the infiltrate was higher in patients with spontaneous regression than in patients for whom no regression occurred. The frequency of T-lymphocytes in the infiltrate around the tumor is therefore considered one of the main causes of spontaneous regression.

Apropos methods for determining the radicality of the surgery, Mohs micrographic surgery can be mentioned $^{8}$. This is based on tumor enucleation using a sharp curette and progressive edge verification of positivity under an operating microscope. Once the tissue samples at the resection margins are negative, the defect can be reconstructed. Mohs micrographic technique has been recommended for areas with high demands on aesthetics such as localized tumors of the head and neck. However, Brisset et al. ${ }^{9}$ point to the lower survival rate of patients treated with Mohs technique compared to patients treated by extensive radical excision supplemented by cervical block dissection.

The basis of successful treatment, however, remains prevention, consisting of regular dermatological check ups in immuno compromised individuals, and early initiation of combination therapy, based on radical surgical treatment, supplemented as needed by radiotherapy and palliative chemotherapy as a last resort.

In the case of the patient described here, given the uncertain prognosis of the disease, it is too early to assess the efficacy of treatment and the situation is complicated by hematological malignancy. However we believe it is useful to publish our experience.

\section{REFERENCES}

1. Hodgson NC. Merkel cell carcinoma: Changing Trends in the incidence. Journal of Surgical Oncology 2005;89(1):1-4.

2. Goepfer H Remmier D, Silva E, Wheller B. Merkel cell carcinoma (endocrine carcinama of the skin) of the Head and Neck. Archives of otolaryngology 1984;110:707-12.

3. Brenner B, Sulkes A, Rakowski E. Second neoplasmas in patiens with Merkel cell carcinoma. Cancer 2001;91:1358-62.

4. Feng $H$, Shude $M$, Chang $Y$. Clonal integration of polyomavirus in human Merkel cell carcinoma. Science 2008;319(5866):1096-100.

6. Mori Y, Hashimoto K, Tanaka K, Cui CY, Mehregan DR, Stiff MA. A study of apoptosis in Merkel cell carcinoma: an immunohistochemical, ultrastructural, DNA ladder, and TUNEL labeling study American Journal of dermathopatology 2001;23:16-23.

7. Inoue T, Yoneda K, Manabi M Demitsu T. Spontaneous regression of Merkel cell carcinoma: a Comparative Study of TUNEL index and tumor-infiltrating lymphocytes Between spontaneous regression and non-regression group. Journal of Dermatological Science 2000;24:203-11.

8. Cottel WI, Bailin PL, Albom MJ, Bernstein G, Braun M, Hanke CW Sutnick TB, Swanson NA. Essentials of Mohs micrographic surgery. Journal of dermatologic surgery and oncology 1988;14:11-3.

9. Brissett $A E$, Olsen KD, Kasperbauer JL, Lewis JE, Goellner JR, Spotts $\mathrm{BE}$, Weaver AL, Strome SE. Merkel cell carcinoma of the Head and Neck: A Retrospective Case Series. Head Neck 2002;24:982-8. doi: 10.1002/hed.10153. 